\title{
Edema de Berlín secundario a Traumatismo ocular cerrado
}

\author{
Berlin Edema secondary to closed eye trauma
}

\author{
Edema de Berlim secundário a trauma ocular fechado
}

\author{
Fermín Silva Cayatopa \\ silvafermin668@gmail.com \\ ORCID: 0000-0002-4033-3163 \\ Robinson Barrientos Ortiz
robinbarrientos07@gmail.com
ORCID: 0000-0002-5085-160k
}

\author{
Ana Luisa González Méndez \\ dra.analuisagonzalez88@gmail.com \\ ORCID: 0000-0002-9821-1345 \\ Gladys Ortiz Saavedra \\ gladysortizsaavedra5@gmail.com \\ ORCID: 0000-0001-8754-3507
}

Clínica La Luz. Lima, Perú

Recibido 14 de octubre 2020 | Arbitrado y aceptado 29 de octubre 2020 | Publicado 22 de diciembre 2020

\section{RESUMEN}

Introducción: El trauma ocular es una de las causas que difieren entre áreas urbanas de un país a otro y entre diferentes clases demográficas o socioeconómicas. Objetivo: Demostrar la evolución y características clínicas del edema de Berlín secundario a trauma ocular cerrado. Materiales y métodos: Se realizó un estudio de caso observacional en un paciente masculino de 11 años que presentó una AVMC 20/20 en OD y cuenta dedos $50 \mathrm{~cm}$ en Ol posterior a trauma ocular cerrado contuso con objeto romo. Desde el inicio desarrolló una conmoción retiniana asociada a edema de Berlín en OI. Con seguimiento desde 11 de diciembre de 2019 al 27 de enero de 2020. Las variables fueron: agudeza visual mejor corregida, retinografía, tomografía de coherencia óptica de dominio espectral macular. Resultados: Se indicó metilprednisolona $500 \mathrm{mg}$ endovenoso diario por 3 días; prednisona $30 \mathrm{mg}$ oral disminuyendo gradualmente durante 10 días; acetato de prednisolona $1 \%$ tópico cada 2 horas, moxifloxacina $0,5 \%$ cada 6 horas, ciclopentolato $1 \%$ cada 8 horas. 7 semanas después, no hubo mejoría clínica a pesar de medicación, manteniéndose en observación médica con AVMC OD 20/20 y Ol cuenta dedos 2 metros. Discusión: Edema de Berlín (commotio retinae) una afección común causada por una lesión contusa en el ojo, suele ser autolimitante y no existe un tratamiento como tal. Conclusión: El trauma ocular cerrado contuso con compromiso retiniano puede causar daño macular como el edema de Berlín, como éste caso que puede condicionar el pronóstico visual a pesar de que mayormente es favorable.

Palabras clave: Trauma ocular cerrado; edema de Berlín; commotio retinae; agudeza visual; tomografía de coherencia óptica

\section{ABSTRACT}

Introduction: Ocular trauma is one of the causes that differ between urban areas from one country to another and between different demographic or socioeconomic classes. Objective: To demonstrate the evolution and clinical characteristics of Berlin edema secondary to closed ocular trauma. Materials and methods: An observational case study was conducted in an 11-year-old male patient who presented a 20/20 BCVA in RE and $50 \mathrm{~cm}$ finger count in LE after blunt ocular trauma with blunt object. From the beginning, she developed a retinal concussion associated with Berlin edema in LE. With follow-up from December 11, 2019 to January 27, 2020. Variables were included: best-corrected visual acuity, retinographies, and macular spectral domain optical coherence tomography. Results: Methylprednisolone $500 \mathrm{mg}$ intravenous daily for 3 days was indicated; prednisone $30 \mathrm{mg}$ oral gradually decreasing over 10 days; $1 \%$ prednisolone acetate topical every 2 hours, moxifloxacin $0.5 \%$ every 6 hours, cyclopentolate $1 \%$ every 8 hours. 7 weeks later, there was no clinical improvement despite medication, and he was kept under medical observation with BCVA RE 20/20 and LE with a 2-meter finger count. Discussion: Berlin edema (commotio retinae), a common condition caused by a blunt injury to the eye, is usually self-limiting and there is no treatment as such. Conclusion: Blunt ocular trauma with retinal involvement can cause macular damage such as Berlin edema, as in this case, which can condition the visual prognosis even though it is mostly favorable.
\end{abstract}

FS: Médico Oftalmólogo Subespecialista en Segmento Anterior, Cornea y Cirugía refractiva. Jefe del servicio de oftalmología del hospital de solidaridad lima peru. Jefe del servicio de oftalmología de las clínicas la luz del Perú. CEO de la clínica la luz. Clínica La Luz. Perú.

AG: Médico Cirujano (2011) egresado de la Universidad Instituto de estudios superiores de Chiapas. México. Fellowship en Investigación en Glaucoma, Oftalmosalud. Especialista En Oftalmología egresado del instituto de ojos Oftalmosalud en Lima- Perú. Fellowship en curso de Segmento Anterior, Cornea y Cirugía Refractiva. Clínica la Luz, Perú. Clínica la Luz. Perú.

RB: Médico Cirujano (2010) egresado de la Universidad Nacional Experimental "Francisco de Miranda" (UNEFM) Venezuela. Especialista En Oftalmología egresado de la Universidad Central de Venezuela. Alta especialidad en Retina y Vítreo egresado del Hospital Dr. Miguel Pérez Carreño, Venezuela y Alta especialidad en Microcirugía del Segmento Anterior - Fundación AVAO Universidad de los Andes (ULA) Venezuela. Clínica La Luz. Perú.

GO: Médico Cirujano egresado de la Universidad del Zulia (LUZ) Venezuela. Especialista En Oftalmología egresado de la Universidad del Zulia (LUZ). Clínica La Luz. Perú.

Key words: Blunt ocular trauma; Berlin edema; commotio retinae; visual acuity; optical coherence tomography 
FS: Médico Oftalmólogo Subespecialista en Segmento Anterior, Cornea y Cirugía refractiva. Jefe del servicio de oftalmología del hospital de solidaridad lima peru. Jefe del servicio de oftalmología de las clínicas la luz del Perú. CEO de la clínica la luz. Clínica La Luz. Perú.

AG: Médico Cirujano (2011) egresado de la Universidad Instituto de estudios superiores de Chiapas. México. Fellowship en Investigación en Glaucoma, Oftalmosalud. Especialista En Oftalmología egresado del instituto de ojos Oftalmosalud en Lima- Perú Fellowship en curso de Segmento Anterior, Cornea y Cirugía Refractiva. Clínica la Luz, Perú. Clínica la Luz. Perú.

RB: Médico Cirujano (2010) egresado de la Universidad Nacional Experimental "Francisco de Miranda" (UNEFM) Venezuela. Especialista En Oftalmología egresado de la Universidad Central de Venezuela. Alta especialidad en Retina y Vítreo egresado del Hospital Dr. Miguel Pérez Carreño, Venezuela y Alta especialidad en Microcirugía de Segmento Anterior - Fundación AVAO Universidad de los Andes (ULA) Venezuela. Clínica La Luz. Perú.

GO: Médico Cirujano egresado de la Universidad del Zulia (LUZ) Venezuela Especialista En Oftalmología egresado de la Universidad del Zulia (LUZ). Clínica La Luz. Perú.

\section{RESUMO}

Introdução: O trauma ocular é uma das causas que difere entre áreas urbanas de um país para outro e entre diferentes classes demográficas ou socioeconômicas. Objetivo: Demonstrar a evolução e as características clínicas do edema de Berlim secundário a trauma ocular fechado. Materiais e métodos: Foi realizado um estudo de caso observacional em um paciente do sexo masculino, 11 anos, que apresentou BCVA 20/20 em OD e contagem de dedos de $50 \mathrm{~cm}$ em OE após trauma ocular fechado contuso com objeto contuso. Desde o início, ela desenvolveu uma concussão retina associada ao edema de Berlim em LE. Com acompanhamento de 11 de dezembro de 2019 a 27 de janeiro de 2020. As variáveis foram: melhor acuidade visual corrigida, retinografias e tomografia de coerência óptica de domínio espectral macular. Resultados: Foi indicada metilprednisolona $500 \mathrm{mg}$ intravenosa ao dia por 3 dias; prednisona $30 \mathrm{mg}$ oral diminuindo gradualmente ao longo de 10 dias; Acetato de prednisolona $1 \%$ tópico a cada 2 horas, moxifloxacina $0,5 \%$ a cada 6 horas, ciclopentolato $1 \%$ a cada 8 horas. 7 semanas depois, não houve melhora clínica apesar da medicação, e ela foi mantida em observação médica com AVMC OD 20/20 e LE com uma contagem de 2 metros nos dedos. Discussão: O edema de Berlim (commotio retinae), uma condição comum causada por uma lesão contusa no olho, geralmente é autolimitado e não há tratamento como tal. Conclusão: O trauma ocular fechado com envolvimento retiniano pode causar danos maculares como o edema de Berlim, como neste caso, que pode condicionar o prognóstico visual, embora seja na maioria favorável.

Palavras-chave: Trauma ocular fechado; edema de Berlin; commotio retinae; acuidade visual; tomografia de coerência óptica

\section{INTRODUCCIÓN}

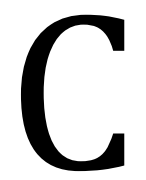

ommotio retinae, también conocido como edema de Berlín, se observa con frecuencia después de un traumatismo ocular cerrado. Se caracteriza por una coloración retiniana grisblanquecina transitoria y una disminución de la agudeza visual mejor corregida (AVMC). Puede estar confinado a la mácula o afectar áreas de la retina periférica (1). Dependiendo de la gravedad del trauma, la histopatología puede revelar áreas de ruptura de la unión del segmento interno/externo, hiperreflectividad de la retina suprayacente, trastornos de la pigmentación y atrofia retiniana (2). Se han propuesto edema extracelular e inflamación glial para la coloración retiniana grisblanquecina transitoria (3). Puede haber lesiones asociadas, como hemorragias retinianas (prerretinianas, intrarretinianas, subretinianas) y ruptura coroidea (4). Los pacientes pueden no tener molestias visuales si sólo está afectada la retina periférica, pero pueden tener una pérdida visual grave cuando hay lesiones maculares (2). Los casos leves presentan pérdida visual transitoria y se recuperan espontáneamente con secuelas mínimas (5), pero los casos más graves pueden asociarse con pérdida visual permanente. $(1,4,6)$. La tomografía de coherencia óptica (OCT) permite la visualización de anomalías estructurales de la mácula y su evolución y sus hallazgos suelen mostrar ruptura de la unión del segmento interno / externo y la correspondiente hiperreflectividad (2), defectos en las puntas del cono OS o daño en la membrana limitante externa (5). Está hipotetizó que el daño severo de los fotorreceptores conduce a peores resultados visuales y anatómicos (5). 


\section{DESCRIPCIÓN DEL CASO CLÍNICO}

$\mathrm{M}$

asculino de 11 años, previamente sano, se presentó a la consulta de emergencia en Clínica la Luz, Lima-Perú. Refiriendo traumatismo contundente en el OI con objeto romo "roca" con disminución de la agudeza visual en el ojo izquierdo (OI) de 4 días de evolución. En la visita inicial, la AVMC en el OD era 20/20 y en el OI cuenta dedos a $50 \mathrm{~cm}$. Sin alteración de los movimientos oculares ni defectos pupilares aferentes. En la biomicroscopía del segmento anterior fue normal en el OD, pero el OI, presentaba equimosis periorbitaria, hiposfagma $360^{\circ}$, córnea transparente no tiñe con fluoresceína, cámara anterior formada, VH 4/4, celularidad +++, atrofia iridiana desde hora 2 a hora 7 con facodonesis, cristalino con esclerosis nuclear grado 1. (Figura 1A) El fondo de ojo: OI mostró una lesión macular central de 1 disco de diámetro con fibrosis, aumento del grosor retiniano y hemorragia intrarretiniana (Figura 1B). El examen de retina periférica fue normal. OCT de dominio espectral, mostró una interrupción de la unión de los segmentos internos y externos de los fotorreceptores con el correspondiente aumento de la reflectividad, pérdida de la capa nuclear externa, infiltración celular de la pared de la retina y fibrosis subretiniana. El grosor de la retina fue de $276 \mu \mathrm{m}$ en el sitio de la lesión (Figura 1C). La angiografía de retina por tomografía de coherencia óptica (Angio- OCT) reveló una zona de baja de correlación y alteración del patrón vascular en la zona foveal central asociado con disrupción del epitelio pigmentario de la retina. Con el apoyo de Pediatría, se realizó una terapia de pulso alto en esteroides fuera de etiqueta (metilprednisolona intravenosa $500 \mathrm{mg}$ durante 3 días y prednisona oral $30 \mathrm{mg}$, disminuyendo gradualmente durante 10 días), lo que resultó en un aumento de la AVMC del OI a cuenta dedos 1 metro y una disminución de espesor retiniano de $51 \mu \mathrm{m}$ a $225 \mu \mathrm{m} 1$ semana después del tratamiento. En un intento de lograr una mejora adicional sin efectos sistémicos no deseados, se continuó con tratamiento vía oral. La AVMC en OI mejoró a cuenta dedos 2 metros, y el grosor de la retina disminuyó en $10 \mu \mathrm{m} 7$ semanas después de dicho tratamiento. 1 mes y medio después de la presentación inicial, la AVMC de OI fue de cuenta dedos 2 metros y el grosor de la retina fue de $215 \mu \mathrm{m}$. Sin embargo, las alteraciones en la fundoscopia y OCT se mantuvieron. (Figura 2 A y B). 

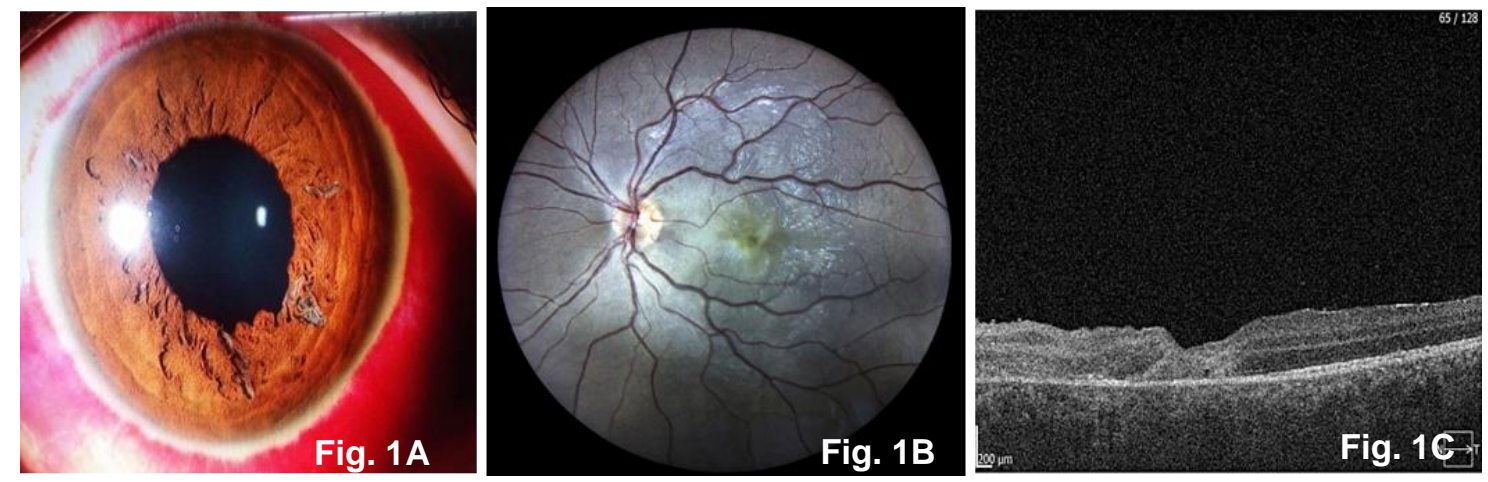

Figura 1. 1A. Retinografía del segmento anterior ocular, que describe hiposfagma $360^{\circ}$, córnea transparente, atrofia iridiana desde hora 2 a hora 7, con esclerosis cristaliniana nuclear grado 1. Figura 1B. Retinografía, revela una lesión macular central de 1 disco de diámetro con fibrosis, y Figura 1C. OCT del OI en el momento de la presentación que muestra rotura de la unión de los segmentos internos y externos de los fotorreceptores con el correspondiente aumento de la reflectividad, infiltración de la pared retiniana y desprendimiento del epitelio pigmentario retiniano. El grosor de la retina fue de $276 \mu \mathrm{m}$ en el sitio de la lesión.
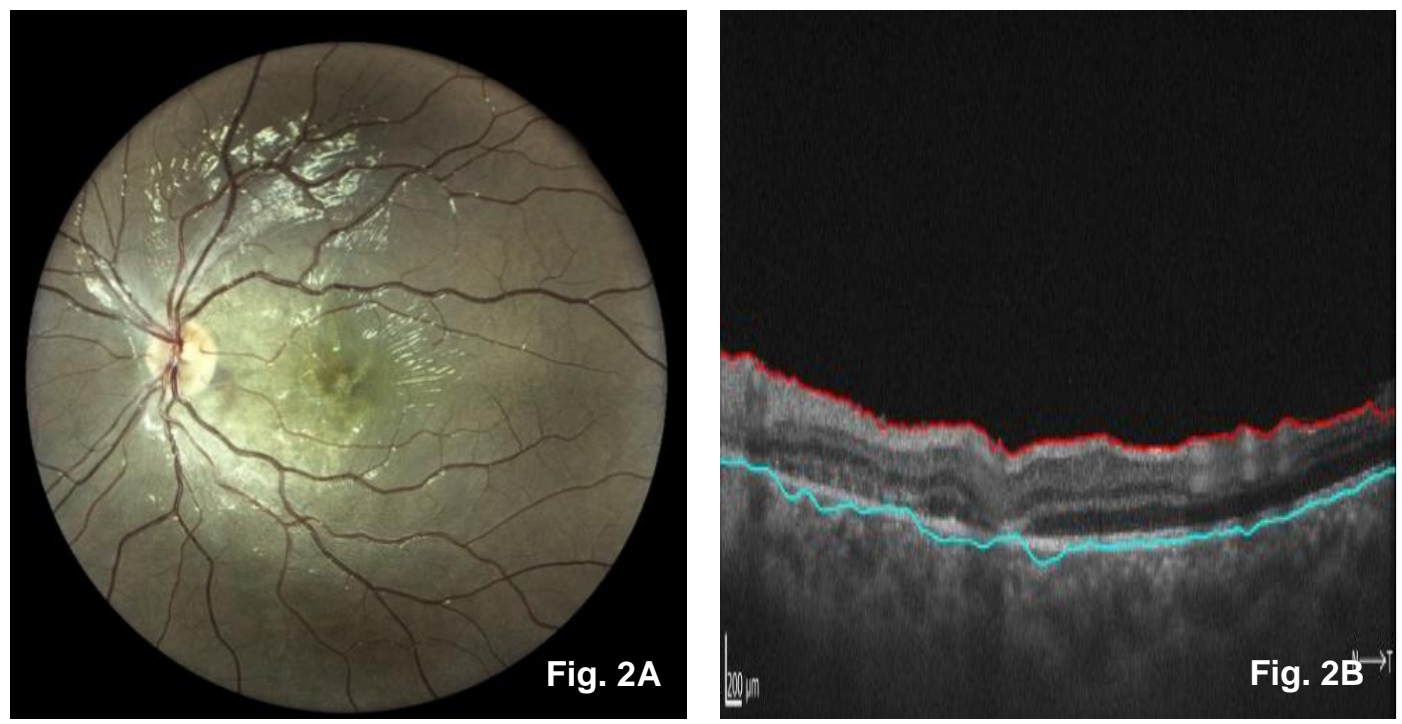

Figura 2. 2A. Retinografía, persiste una lesión macular central de 1 disco de diámetro con fibrosis;

Figura. 2B. Último OCT del OI 7 semanas después de la presentación inicial, que muestra una disminución del grosor retiniano $(215 \mu \mathrm{m})$. 


\section{DISCUSIÓN}

$\mathrm{E}$ 1 daño del segmento posterior después de un traumatismo cerrado coexiste con frecuencia con el daño del segmento anterior al igual que lo reportado en éste caso 1 . Los pacientes con una unión de los segmentos internos y externos de los fotorreceptores dañada tienen más probabilidades de tener defectos fotorreceptores persistentes, con pérdida irreversible de fotorreceptores 2,5. En nuestro caso, el desarrollo de fibrosis, alteraciones en la unión de segmento interno y externo y pérdida de la capa nuclear externa que se evidenció en la OCT, limitó la recuperación de la lesión retiniana permaneciendo sin tratamiento durante un largo período.

A partir de la Angio- OCT, los autores concluyeron que hubo una zona de baja de correlación y alteración del patrón vascular en la zona foveal central asociado con disrupción del epitelio pigmentario de la retina (Figura 2). Por tanto, el diagnóstico fue de lesión fibrótica y no de membrana neovascular. La OCT mostró un aumento del grosor de la retina, probablemente debido a un edema inflamatorio postraumático. Con el apoyo de Pediatría, se realizó una terapia de pulsos de esteroides fuera de etiqueta en dosis altas (metilprednisolona intravenosa $500 \mathrm{mg}$ durante 3 días y prednisona oral $30 \mathrm{mg}$, disminuyendo gradualmente durante 10 días), lo que resultó en un aumento de la AVMC del OI a cuenta dedos 1 metro y una disminución del grosor de la retina de $51 \mu \mathrm{m}$ a $225 \mu \mathrm{m} 1$ semana después del tratamiento. En un intento de lograr una mejora adicional sin efectos sistémicos no deseados, se continuó con tratamiento vía oral.

La AVMC en OI mejoró a cuenta dedos 2 metros, y el grosor de la retina disminuyó en $10 \mu \mathrm{m} 7$ semanas después de dicho tratamiento. 1 mes después de la presentación inicial, la AVMC de OI fue de cuenta dedos 2 metros y el grosor de la retina fue de $215 \mu \mathrm{m}$. Lo más probable es que la recuperación se deba a la terapia, ya que 1 mes después de la lesión inicial no se esperaba una recuperación espontánea. El hecho de que éste paciente haya sido seguido durante 4 semanas después del tratamiento y que la condición no mejorará parece indicar una lesión fibrótica más que una membrana neovascular. Se realizó una búsqueda en PubMed utilizando los siguientes términos: commotio retinae y tratamiento.

Hasta donde se sabe, son pocos los artículos publicados previamente con tal retraso en el tratamiento y el uso de terapia de pulsos de esteroides en dosis altas fuera de etiqueta en un joven.

Este caso parece indicar que vale la pena tratar las lesiones por traumatismo sin resolución espontánea, incluso cuando han progresado hasta por 7 semanas. Sin embargo, cuando ocurre un daño estructural, la recuperación puede ser limitada.

- Conflicto de intereses. Sin conflicto de intereses

- Financiación. Sin financiamiento

- Agradecimiento: Especial agradecimiento a Clínica La Luz en Lima, Perú. Al jefe del departamento de Segmento Anterior, Córnea y Cirugía Refractiva. Dr. Fermín Silva por permitir la realización de éste trabajo.

- Revisión realizada considerando los tratados bioéticos

\section{REFERENCIAS BIBLIOGRÁFICAS}

1. Blanch RJ, Good PA, Shah P, Bishop JRB, Logan A, Scott RAH. Visual outcomes after blunt ocular trauma. 
Ophthalmology. Agosto de 2013;120(8):1588-91

2. Souza-Santos F, Lavinsky D, Moraes NS, Castro AR, Cardillo JA, Farah ME. Spectral-domain optical coherence tomography in patients with commotio retinae. Retina. Abril de 2012;32(4):711-8

3. American Academy of Ophthalmology, The Eye M.D. Association: Posterior segment manifestations of trauma; in Basic and Clinical Science Course, section 13: Retina and Vitreous. Leo, 20112012

4. Moon K, Kim KS, Kim YC. A case of expansion of traumatic choroidal rupture with delayed-developed outer retinal changes. Case Rep Ophthalmology. 2013;4(2):70-5

5. Ahn SJ, Woo SJ, Kim KE, Jo DH, Ahn J, Park KH. Optical coherence tomography morphologic grading of macular commotio retinae and its association with anatomic and visual outcomes. Am J Ophthalmol. Noviembre de 2013;156(5):9941001.e1

6. Saleh M, Letsch J, Bourcier T, Munsch C, Speeg-Schatz C, Gaucher D. Longterm outcomes of acute traumatic maculopathy. Retina. Noviembre de 2011;31(10):2037-43 